\title{
BROWSERLESS WEB AUTOMATION FOR BLIND USERS
}

\author{
Ruslan R. Fayzrakhmanov and Andrey Kravchenko \\ Department of Computer Science, University of Oxford, UK
}

\begin{abstract}
Navigating and performing different tasks on the Web can be frustrating for blind users. This is related to different aspects including a linear nature of navigation through the content, the issues of contemporary web pages not following accessibility guidelines, and limitations of assistive technologies trying to keep pace with a constantly changing web stack of technologies. Web automation tools help users to automate tasks and ease the navigation to a desired content. However, most of existing approaches are based on the web browser and, therefore, have some requirements on the hardware and software used, its performance. Furthermore, they have to take into account complex sequences of DOM events which reflect different changes and states on a web page, such as completion of the content loading or reaction on user actions. These non-trivial conglomerates of events often considerably reduce the robustness of web automation. In this paper we introduce a new approach, FastWrap4A, which can analyse user interactions and transpose the automation from the UI level directly to the level of data-focused interactions with web servers. This approach does not require a browser or an integrated web page rendering engine and can be executed on different devices.
\end{abstract}

\section{KEYWORDS}

Web Accessibility, Web Automation, Assistive Technology

\section{INTRODUCTION}

The Web is a huge information platform that provides vast opportunities for people by making it possible to effectively solve various tasks in business, education, science, and our everyday lives. However, these opportunities are not available for everybody. For example, people who are blind encounter a lot of problems surfing the Web. This is a direct result of the majority of web pages not following accessibility guidelines and standards, being oriented at sighted users, and not taking into account users who are blind. Moreover, web accessibility standards unfortunately always tend to be a step behind rapidly changing technologies and cannot cover various aspects of the web design and web navigation (Nogueira et al. 2019, Power et al. 2012).

There are two main directions for approaching the enhancement of web accessibility and usability: (1) by means of developing standards and guidelines which lead to the creation of more accessible content for specific groups of users and corresponding assistive technology that provides easier access to the accessible content; (2) by means of developing approaches and tools for making inaccessible content accessible (Fayzrakhmanov, 2013). Web automation is one of means to enhance accessibility of inaccessible websites. It is a technology that automates typical web browsing activities such as form filling and clicking links to reach the required content, by performing these actions on behalf of the user. Web automation is usually performed by manual invocation of a desired script, presented as a sequence of actions. A script can be written manually or automatically derived, for instance, with a technique such as programming by example (PBE).

Some representative examples of web automation systems are CoScripter (Leshed et al. 2008) (PBE-based system which enables users to record, play back, edit, and share generated scripts; the latter are both human- and machine-understandable), TrailBlazer (Bigham et al. 2009) (it assists blind users in performing certain web-based tasks while dynamically generating non-visual interface based on the database of scripts provided by CoScripter, and a relevant script is suggested to the user based on a provided short task description), Ubiquity (Erlewine 2009) (a multilingual textual interface for the Firefox browser enabling rapid information retrieval and task execution), CoCo (Lau et al. 2010) (framework which provides the user with necessary scripts with short textual commands and assists her with web tasks, utilising ActionShot and shared database of CoScripter), Ringer (Shaon et al. 2016) (a heuristic approach to browser-based PBE). 
Most of known web automation systems are based on a web browser, the analysis of rendered web pages and mimicking user actions. Due to the complexity of contemporary web applications the use of the rendering engine is essential, since a web page can be constructed from different sources integrated via AJAX requests and transformed with comprehensive JavaScript code. The rendering typically consists of (a) parsing the web page and building a DOM tree, (b) applying CSS rules, fonts, and (c) executing JavaScript, which is usually the most computationally expensive part, often having wide-ranging effects on the page's DOM. The rendering can thus take up to $98 \%$ of the runtime with about $2 \%$ for actual data analysis and extraction (Furche et al., 2013). Another complicated problem of browser-based approaches is their robustness. They have to deal with the analysis of complex sequences of DOM events reflecting different states of a web page (e.g., load, DOMContentLoaded, unload, change, DOMNodelnserted) and handling different tabs opened after simulating user clicks. For example, OXPath implements non-trivial algorithms for handling complex cases related to changes on a web page caused by simulated user interaction and distinguishing them from those (irrelevant) changes related to updates in news or ads blocks.

In this paper, we present FastWrap4A, a best-effort solution to eliminate the web browser completely and provide the blind user with a more efficient and robust record-and-extract automation. Omitting the web browser gives us the possibility to directly interact with relevant web servers on the level of HTTP interactions, issuing only relevant HTTP requests. The goal of our invention is thus not related to the automation of user interactions with the user interface, but rather automation of the data access, making it robust and accessible. The core technology, FastWrap (Fayzrakhmanov et al. 2018), demonstrates its high robustness and performance. FastWrap scripts run, on average, in less than $7.14 \%$ of the runtime of the state-of-the-art web data extraction programs, using $1.77 \%$ of the HTTP calls, and $3.12 \%$ of the Web traffic. The scripts keep precision and recall equal to those of traditional web data extraction scripts (so-called wrappers).

FastWrap4A can generate parameterised automation scripts either in a PBE fashion with the use of a web browser extension FastWrap4A Listener or directly from the analysis of existing automation scripts, such as Ringer (Shaon et al. 2016). FastWrap4A Listener can be used by an experienced blind user or a sighted user to let FastWrap4A mine patterns within HTTP interactions and generate a script. This script models specific type of user tasks, be it checking the exchange rate for different currencies or train schedule for arbitrary locations. In this examples, currencies and locations are input parameters which are to be provided by the blind user while running the generated script. A direct generation of the FastWrap4A script is also possible by executing an existing script produced by another system. FastWrap4A can thus build its automation script either from HAR file or by listening to the HTTP request-response exchanges via the proxy server.

\section{ARCHITECTURE AND APPROACH}

FastWrap4A consists of two main modules: FastWrap4A Listener (a browser extension for Chrome for gathering HTTP interactions and the user input-output) and FastWrap4A Engine (a FastWrap-based technology for generating and running an automation script).

When the user surfs the Web, the Listener collects data-rich interactions between the client software and web servers along with entered data (e.g., via form filling) and the desired output. The input can be, for example, departure-arrival locations and dates in case of flight search or location in case of property search, while the output can be available flights or properties along with their prices. The Listener can be implemented as a proxy server, collecting all necessary HTTP interactions, or an extension for the web browser. In this paper we implemented FastWrap4A Listener realised as a browser extension as illustrated in Figure 1, which is compatible with popular screen readers, such as JAWS and NVDA. With the use of a preferable assistive technology and the FastWrap4A browser extension, the user can start a recording session navigating to a desired web page, entering data into web forms, performing interactions to navigate to a resulting page containing desired data. When the session is complete and desired data is labelled with the use of the browser extension, generated data-rich HTTP traces with input-output annotations are saved on the file system. Our study shows that usually three repetitions of the same interaction scenario with different input data are enough to construct a robust automation script.

Given a set of recorded HTTP traces (with input and output data), FastWrap4A Engine analyses them and generates a script which automates this scenario. The resulting script does not need a web browser and can be directly executed on any device, be it a PC or a mobile phone (Figure 1). It directly interacts with servers 
issuing only those HTTP requests which are related to obtaining desired data. To run a specific recorded scenario, it is enough to provide input data, usually the data entered in a web form, via an accessible web interface. Extracted data is returned as a table with ARIA annotations.
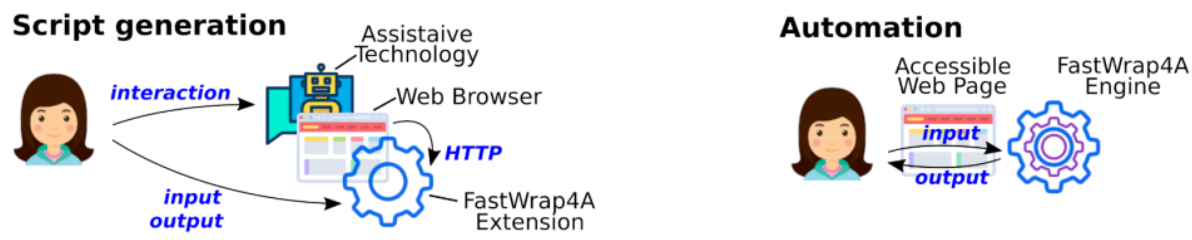

Figure 1. Software architecture for script generation and automation with FastWrap4A

Thus, through using our automation technology the user does not face the overhead related to web browser initialisation and rendering, which considerably increases the robustness and decrease the runtime of an automated scenario.

\section{AUTOMATION WITH FASTWRAP4A}

In this section, we introduce FastWrap4A Engine, our approach for automatically generating and executing HTTP scripts for web automation. A detailed description of the base approach with comprehensive evaluation is presented in (Fayzrakhmanov et al. 2018).

FastWrap4A Engine takes as input collected HTTP interactions (e.g., from the browser extension, web logs or an HAR file), along with input and output data, and produces as output a corresponding browserless HTTP script. The script extracts desired data by directly interacting with the website's servers and does not require loading and rendering the web page. Thus, it is more portable than existing automation technologies and also more robust.

The FastWrap4A Engine analyses the sequence of HTTP request-response exchanges (referred to as HTTP traces) between the client (i.e. a web browser or page rendering engine) and web servers. HTTP traces also include desired data labelled by the user (so-called output records). This data is used to identify final HTTP interactions with the desired output records in their responses. Furthermore, the provided records are used to generate data selectors to extract the data from the content and transform this data into a structured representation. If input parameters are necessary (e.g., for web form filling), HTTP traces also include these. Input and output parameters are used to correctly induce the output script and guarantee that the desired data is not overlooked. Automation of script creation is thus solely based on the direct analysis of these HTTP traces. This makes our approach applicable to a wide range of automation scenarios and avoids tying it to any particular system. Our pilot studies also show that it can be applied for transforming existing scripts such as Ringer and OXPath into considerably more efficient scripts, about 24 times faster (Fayzrakhmanov et al. 2018).

The overall FastWrap4A approach can be intuitively explained via the following two main phases.

Phase 1: Given a set of HTTP traces, identify final interactions carrying desired output data and model them as abstract final interactions. Generate data selectors and value transformers.

This step is illustrated in Figure 2. FastWrap4A iterates over all recorded HTTP interactions and detects those which contain the output data selected by the user within the web browser. These final interactions are clustered into sets of interactions according to the similarity of their HTTP response contents and HTTP requests. Each cluster is modelled by a so-called abstract final interaction. It, in turn, defines input parameters necessary to instantiate this class of interactions as well as data selectors to pull the desired user output data from the HTTP response and value transformers to convert extracted data into the necessary form. Both data selectors and value transformers mimic some typical data manipulation and transformation techniques implemented by the web page's JavaScript.

For output data and parameter value extraction from the HTTP response content, FastWrap4A can induce two types of data selectors: XPath for X/HTML documents and JSONPath for JSON(P). All data selectors can also select sub-structures (i.e., subtrees in the case of XPath and nested objects in the case of JSONPath) 
To transform the extracted data into a parameter of a dependent HTTP request or the final output, the following value transformations are supported: (1) string manipulations, such as a subset, removal of double spaces, inserting a constant string, a character replacement; (2) URL encoding and decoding; (3) replacement of HTML escape symbols; (4) numerical manipulations such as changing the precision of real numbers. Our approach identifies the most specific transformation and can combine different transformations with each other, which we find quite useful in practice. For example, space-to-plus conversion followed by URL encoding or URL encoding followed by string concatenation.

(Recursive) Phase 2: Given an abstract interaction, identify other HTTP interactions within the set of HTTP traces containing data in their responses that are necessary to initialise the current abstract interaction. (Instantiation is replacing parameters in an HTTP request of the abstract interaction with relevant data.) Model discovered HTTP interactions as abstract interactions, set a corresponding dependency relation with data selectors and value transformers.

Repeat this step recursively until all dependencies are resolved.

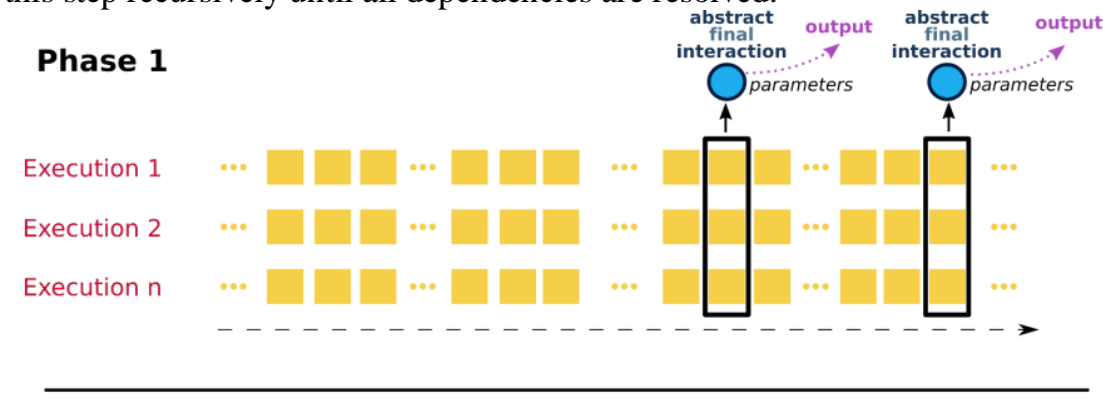

\section{Phase 2}
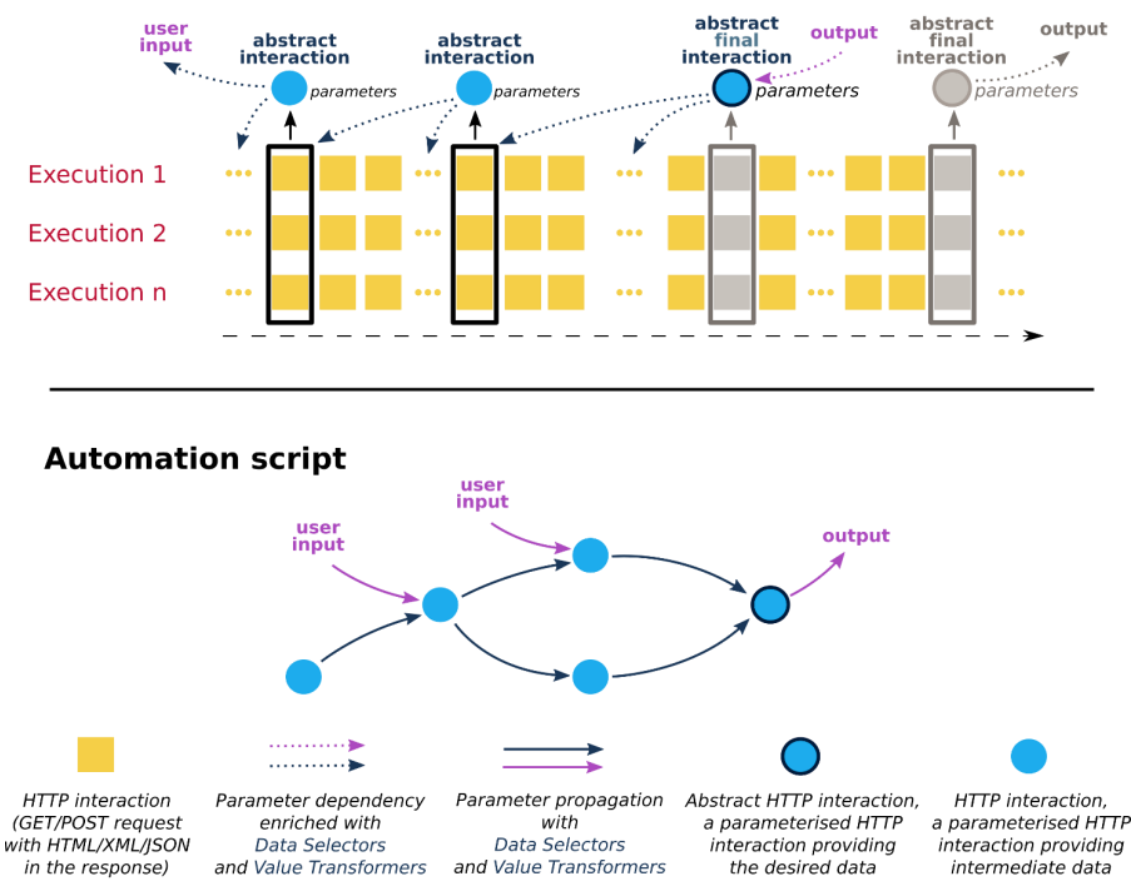

Figure 2. Two phases of automating script generation and schematic representation of the output script

This step is illustrated in Figure 2. Abstract (final) interactions can have input parameters which are propagated from HTTP responses of other interactions. Thus, in this step, FastWrap4A identifies HTTP interactions carrying the desired parameters, clusters those interactions, and generates abstract interactions for each formed cluster, similar to Phase 1 . This phase has a recursive nature since a new-built abstract interaction can also have input parameters. During Phases 1 and 2 a graph is constructed, in which each node represents 
an abstract (final) interaction and arcs reflect the parameter propagation from HTTP interactions providing necessary data in their response to HTTP requests of dependent abstract interactions. Each arc is annotated with corresponding data selectors and data transformers. In case all dependencies are resolved a resulting web automation script is constructed as illustrated in Figure 2.

Automation is the execution of the generated script which requires the user to provide necessary input data, which she enters in the corresponding web form.

The script is executed in a recursive fashion navigating through the graph structure from the abstract final interaction to dependency-resolved interactions, which either require or do not require user input. An abstract interaction is instantiated and triggered in case all its dependent input parameters are instantiated via data selectors and value transformers.

\section{CONCLUSION}

We introduce FastWrap4A, a web automation tool, which can help blind users to automate their web tasks, be it checking flights, prices for flats, or weather forecasts. This approach does not require a web browser and can run on different devices, be it a PC or a mobile phone. Our approach can be used both for automating user interactions and converting existing databases of browser-dependant scripts into more efficient and lightweight automation scripts (e.g., OXPath or Ringer), which, in turn, can directly issue only relevant HTTP interactions providing the user efficiently with desired information in an accessible web content. We believe this novel approach and study can bring new technology and thoughts towards more advanced and robust web automation to blind users. Moreover, as future work we believe it is important to be able to automatically identify interesting information which could be relevant to the user, without requiring her to label the output data.

\section{ACKNOWLEDGEMENT}

This work is supported by the EPSRC programme grant EP/M025268/1 (VADA).

\section{REFERENCES}

Bigham, J. P. et al, 2009. TrailBlazer: Enabling Blind Users to Blaze Trails through the Web. Proceedings of the 14th international conference on Intelligent user interfaces, New York, USA, pp. 177-86.

Erlewine, M. Y., 2009. Ubiquity: Designing a Multilingual Natural Language Interface Features of a Natural Syntax. SIGIR Workshop on Information Access in a Multilingual World, Boston, USA, pp. 45-48.

Fayzrakhmanov, R. R. 2013. Web Accessibility for the Blind Through Visual Representation Analysis. Vienna University of Technology (PhD Thesis), Vienna, Austria.

Fayzrakhmanov, R. R. et al, 2018. Browserless Web Data Extraction: Challenges and Opportunities. Proceedings of the 2018 World Wide Web Conference on World Wide Web (WWW'18). Lyon, France, pp. 1095-1104.

Furche, T. et al, 2013. OXPath: A language for scalable data extraction, automation, and crawling on the deep web. VLDB Journal, Vol. 22, No. 1, pp. 47-72.

Lau, T. et al, 2010. A conversational interface to web automation. Proceedings of the 23nd annual ACM symposium on User interface software and technology. New York, USA, pp. 229-238.

Leshed, G. et al, 2008. CoScripter: Automating \& Sharing How-To Knowledge in the Enterprise. Proceedings of the SIGCHI Conference on Human Factors in Computing Systems, Florence, Italy, pp. 1719-1728.

Nogueira, T. C. et al. 2019. Comparing Sighted and Blind Users Task Performance in Responsive and Non-Responsive Web Design. Knowledge and Information Systems, Vol. 58, No. 2, pp. 319-39.

Power, C. et al. 2012. Guidelines are only half of the story: accessibility problems encountered by blind users on the Web. Proceedings of the SIGCHI Conference on Human Factors in Computing Systems, Austin, Texas, USA, pp. $433-442$.

Shaon, B. et al, 2016. Ringer: web automation by demonstration. Proceedings of the ACM International Conference on Object-Oriented Programming, Systems, Languages, and Applications, Amsterdam, Netherlands, pp. 748-764. 